\title{
Security of a Privacy-Preserving Biometric Authentication Protocol Revisited
}

\author{
Aysajan Abidin $^{1}$, Kanta Matsuura ${ }^{2}$, Aikaterini Mitrokotsa ${ }^{1}$ \\ ${ }^{1}$ Chalmers University of Technology, Gothenburg, Sweden \\ \{aysajan.abidin, aikaterini.mitrokotsa\}@chalmers.se \\ ${ }^{2}$ University of Tokyo, Japan \\ kanta@iis.u-tokyo.ac.jp
}

\begin{abstract}
Biometric authentication establishes the identity of an individual based on biometric templates (e.g. fingerprints, retina scans etc.). Although biometric authentication has important advantages and many applications, it also raises serious security and privacy concerns. Here, we investigate a biometric authentication protocol that has been proposed by Bringer et al. and adopts a distributed architecture (i.e. multiple entities are involved in the authentication process). This protocol was proven to be secure and privacy-preserving in the honest-but-curious (or passive) attack model. We present an attack algorithm that can be employed to mount a number of attacks on the protocol under investigation. We then propose an improved version of the Bringer et al. protocol that is secure in the malicious (or active) insider attack model and has forward security.
\end{abstract}

Key words: Biometrics, privacy-preserving biometric authentication, homomorphic encryption, active attack, forward security.

\section{Introduction}

Biometric authentication offers important advantages mainly due to the uniqueness of biometric identifiers and other favorable properties since biometrics cannot be lost or forgotten. A biometric authentication system consists of two phases, namely, the enrollment phase and the authentication phase; and it typically involves two entities: a client and a server. During the enrollment phase, the client provides the server with his biometric data for storage in a database. Then, during the authentication phase, the server authenticates the client if his fresh biometric template matches the one that is stored in the database.

Since the server often has to perform many tasks (e.g. retrieving from the database the client's reference biometric template, checking if it matches the fresh template) its role can be divided into several parts. Thus, the execution of the protocol involves different entities where each 
entity performs a specific task. For instance, a biometric authentication protocol could involve the following entities: a user $\mathcal{U}$, a biometric sensor $\mathcal{S}$, an authentication server $\mathcal{A S}$, a database $\mathcal{D B}$ and a matcher $\mathcal{M}$. This architecture of a biometric authentication system has been proposed by Bringer et al. [1]. In this new setup, a biometric authentication system works as follows. Let $N$ be the number of users registered in the authentication system. We denote by $\mathcal{U}_{i}$ the $i$-th user where $1 \leq i \leq N$. In the enrolment phase the user $\mathcal{U}_{i}$ registers his biometric data $b_{i}$ which is then stored in the database $\mathcal{D B}$. In the authentication phase a user $\mathcal{U}_{i}$ first provides a fresh biometric trait $b_{i}^{\prime}$ and his identity $\mathrm{ID}_{i}$ to the sensor $\mathcal{S}$, which in turn forwards these data to the authentication server $\mathcal{A S} . \mathcal{A S}$ then asks $\mathcal{D B}$ for $\mathcal{U}_{i}$ 's biometric data $b_{i}$ that is already stored in $\mathcal{D B}$. After getting $b_{i}$ from $\mathcal{D B}, \mathcal{A S}$ sends $b_{i}$ and $b_{i}^{\prime}$ to the matcher $\mathcal{M}$, which checks whether $b_{i}$ and $b_{i}^{\prime}$ match and sends back the result of the comparison to $\mathcal{A S}$, which then makes the decision of whether to grant authentication to the user depending on the matcher's response.

Note that it is assumed that the output of the authentication process denoted as Out $_{\mathcal{A S}}$ (i.e. knowing whether the authentication has been granted or not) is publicly available; something that is quite common in the literature 2 3 4 456]. For instance, in case the biometric authentication system is used to restrict access to a building then the event that the door opens corresponds to a successful authentication.

However, biometric authentication has also many serious security and privacy implications. Compromised biometric templates may lead to serious threats to identity, while the inherent irrevocability of biometrics renders this risk even more serious. Furthermore, biometric information may reveal very sensitive and private information such as genetic [7] and medical information [8]. Additional issues of linkability, profiling and tracking of individuals are raised by cross-matching biometric traits. Therefore, privacy-preserving biometric authentication protocols are of utmost importance. Many existing protocols rely on the use of secure multi-party computation techniques including homomorphic encryption [9] and oblivious transfer [10]11].

Contributions and Related Work In this paper, we review a privacypreserving biometric authentication protocol that has been proposed by Bringer et al. [1. This protocol relies on the Goldwasser-Micali (GM) cryptosystem [12] which is a homomorphic encryption. Bringer et al. [1] have shown that their protocol is secure under the assumption that the system entities do not collude and are honest-but-curious. Here, we improve upon the original protocol to safeguard it against malicious insider attacks. 
We first present a generic algorithm that can be employed by an adversary to mount a number of attacks to the protocol under investigation. One of the enablers of the attacks is the bit-by-bit encryption of the biometric data using the GM encryption scheme. Then, we propose an improved protocol that is secure and privacy-preserving in the malicious adversarial model. In particular, the improved protocol is secure against malicious, but non-colluding insider attacks and has forward security. We also compare our protocol with the original protocol.

Some attacks on the protocol under study were presented by Barbosa et al. [13] and Simoens et al. [14. Barbosa et al. [13] present a simple attack that allows the authentication server $\mathcal{A S}$ to learn some bits of the reference biometric templates due to non-randomisation of the response by the database $\mathcal{D B}$ to the authentication server $\mathcal{A S}$. Simoens et al. [14] present possible insider attack ideas and attacks by a single or multiple, colluding malicious entities. In this paper, we extend some of their attack ideas and present a simple yet powerful attack algorithm.

Bringer and Chabanne [15] presented an improvement of the protocol under study, where they replaced the matching algorithm by an error correction procedure using secure sketches and discussed how it can be integrated into the Private Information Retrieval (PIR) scheme due to Lipmaa [16]. In their scheme, the database stores encryptions of the biometric templates. However, this scheme is computationally expensive. There are also several biometric authentication protocols proposed by Stoianov [17] that employ the Blum-Goldwasser (BG) [18 19] encryption scheme. But in these protocols, there are three entities, namely a client, a computation server (or database), and an authentication server. There are many other works related to privacy-preserving biometrics. However, to the best of our knowledge, Barbosa et al. [13] and Simoens et al. [14] are the only ones that study the security of the protocol under investigation.

Outline After giving some definitions and our threat model in Section 2, we present the protocol under study in Section 3. Then, in Section 4, we describe the attack algorithm. Section 5 presents an improvement of the Bringer et al. protocol while Section 6 presents its security analysis and compares it with the original protocol. Finally, Section 7 concludes the paper and highlights some future work.

\section{Preliminaries}

We give notations and definitions of some of the key concepts used throughout the paper. Also, we present a threat model in which we analyse the security and privacy of the biometric authentication protocol under study. 
Communication Model In our modifications to the protocol under investigation, we assume that there is a secure and authentic channel between the system entities. In particular, we assume that there are shared secret keys between $\mathcal{S}$ and $\mathcal{M}, \mathcal{A S}$ and $\mathcal{M}, \mathcal{D B}$ and $\mathcal{M}$, that are used to encrypt and authenticate messages sent to $\mathcal{M}$. In addition, $\mathcal{M}$ has a public encryption key to which all other system entities have access, and $\mathcal{S}$ and $\mathcal{D B}$ have a shared secret key that they use to derive a permutation to permute the biometric templates before encrypting them. Since we omit the underlying infrastructure for the public-key primitive (i.e. the protocol does not explicitly use certificates), we also assume the authenticity of the matcher's public key. In this paper, we focus on the case where there is only a single $\mathcal{S}$, a single $\mathcal{A S}$, and a single $\mathcal{D B}$ in the system. Therefore, security in the case of multiple entities communicating with each other in parallel is outside the scope of this paper.

Definitions We use the following as a definition of privacy-preserving biometric authentication.

Definition 1 (Privacy-preserving biometric authentication). We say a biometric authentication protocol is privacy-preserving if no probabilistic polynomial-time (PPT) adversary can recover any of the following information, if they are not already known: a fresh biometric $b_{i}^{\prime}$, a stored biometric $b_{i}$ or the correspondence between the identity $I D_{i}$ and $b_{i}$.

We also use provably secure message authentication codes (MACs) in our modification to the protocol under study. A MAC scheme MAC consists of a key generation algorithm KeyGen, a tag generation algorithm TAG, and a verification algorithm VRFY. When we say a MAC scheme is $\epsilon$-secure, we refer to the following definition.

Definition 2. A MAC scheme is called $\epsilon$-secure if no PPT adversary $\mathcal{A}$ can generate a valid message-tag pair, even after making polynomially many tag generation and verification queries, except with probability $\epsilon$.

Furthermore, when we say secure pseudorandom number generator (PNG) we mean a PNG that satisfies the following definition.

Definition 3. A PNG is called an $\epsilon$-secure if no PPT distinguisher $D$ can distinguish its output from a randomly chosen bitstring of equal length except for a negligible probability $\epsilon$.

Lastly, we use symmetric key encryption, denoted by SKE, in our modification. We require SKE to have indistinguishability against ciphertext-only attacks (IND-COA) (cf. Appendix A). Note that we use Enc (and Dec) to 
denote the GM encryption (and decryption), and $\mathrm{Enc}_{K}$ (and $\mathrm{Dec}_{K}$ ) to denote symmetric key encryption (and decryption) with a key $K$.

Threat Model In our threat model, we go beyond the honest-but-curious (or passive) model that is adopted in the original protocol by Bringer et al. 1 and extend the adversary model investigated by Simoens et al. [14. Hence, we consider as an adversary $\mathcal{A}$ any passive (or active) internal entity that can violate the protocol specifications and that attempts to recover any of the following information, if they are not yet known: the fresh biometric $b_{i}^{\prime}$, the stored template $b_{i}$, and/or the correspondence of a user identity $\mathrm{ID}_{i}$ to the stored template $b_{i}$. Thus, each of the entities - the user $\mathrm{ID}_{i}$, the sensor $\mathcal{S}$, the authentication server $\mathcal{A S}$, the database $\mathcal{D} \mathcal{B}$, and the matcher $\mathcal{M}$ - may pose threats to privacy of biometric reference, biometric sample and user identity [14].

Assumptions When security and privacy of a biometric authentication system are analysed, there are always certain assumptions that must hold. In our case, we make the following assumptions.

Assumption 1 We assume that the sensor $\mathcal{S}$ is honest, has not been compromised and captures the biometric templates from alive human users.

This assumption is important because if the sensor $\mathcal{S}$ is compromised, then the adversary can wait until a legitimate user comes and authenticates himself to the system, and hence easily learns the identity and fresh biometric template of a legitimate user. This is possible because as we mentioned earlier the output of the authentication server to the user is assumed to be publicly known.

A malicious user may attempt to get himself authenticated to the system by a fake identity and a fake biometric template. Also, a series of successful consecutive authentication attempts by the same user identity may also be an indication of a malicious behaviour if there is a specific pattern in the biometric templates used. Therefore, we assume that the system has appropriate measures to limit the number of such trials. This brings us to our next assumption.

Assumption 2 We assume that the biometric authentication system has a limit on the maximum allowed consecutive failed trials to grant access. This limit does not allow an adversary to create a fake fresh biometric $b_{i}^{\prime}$ that is accepted by the matcher $\mathcal{M}$. Also, we assume that the system has a limit on the maximum allowed consecutive successful trials to grant access. This limit helps the system to detect hill climbing attacks; see Simoens et al. 14] for details on this attack. 
Finally, we assume that the system entities are not colluding. We note that this assumption is valid when an adversary has compromised only one of the entities. And we believe that this is an important first step towards achieving a protocol secure against malicious and colluding insider attacks.

Assumption 3 We assume that the entities $\mathcal{A S}, \mathcal{D B}, \mathcal{M}$ may not collude with each other.

\section{The Bringer et al. Protocol}

Bringer et al. [1] have proposed a protocol for privacy-preserving biometric authentication that follows the above described model and involves four entities in the biometric authentication process. According to this protocol the sensor $\mathcal{S}$, the authentication server $\mathcal{A S}$ and the database $\mathcal{D B}$ store the public key pk while the matcher $\mathcal{M}$ stores the secret key sk. $\mathcal{A S}$ also stores the mapping $\left(\mathrm{ID}_{i}, i\right)$, for $i=1, \ldots, N$, where $i$ corresponds to user $\mathcal{U}_{i}$ and $N$ is the total number of users of the biometric authentication system. Furthermore, $\mathcal{D B}$ stores the reference biometric template $b_{i}$. The protocol is based on the GM cryptosystem. We denote by $\operatorname{Enc}\left(b_{i}\right)$ the bit-by-bit encryption of the template $b_{i}$, i.e. $\operatorname{Enc}\left(b_{i, 1} \ldots b_{i, M}\right)=\left(\operatorname{Enc}\left(b_{i, 1}\right), \ldots, \operatorname{Enc}\left(b_{i, M}\right)\right)$, where $M$ is the bit length of the template.

In the enrolment phase, user $\mathcal{U}_{i}$ registers $\left(b_{i}, i\right)$ at $\mathcal{D B}$, and $\left(\mathrm{ID}_{i}, i\right)$ at the $\mathcal{A S}$. The authentication phase comprises the following phases.

Phase 1 - COMmunication $\mathcal{U}_{i} \rightarrow \mathcal{S} \rightarrow \mathcal{A S}$ :

- $\mathcal{U}_{i}$ provides a fresh biometric trait $b_{i}^{\prime}$ and his identity $\mathrm{ID}_{i}$ to $\mathcal{S}$.

- Then, $\mathcal{S}$ sends the fresh biometric $b_{i}^{\prime}$ encrypted under the public key pk (i.e. $\left.\operatorname{Enc}\left(b_{i}^{\prime}\right)\right)$ as well as the claimed identity $\mathrm{ID}_{i}$ to $\mathcal{A S}$.

Phase 2 - Communication $\mathcal{A S} \leftrightarrow \mathcal{D} \mathcal{B}$ :

- $\mathcal{A S}$ performs the mapping from $\mathrm{ID}_{i}$ to $i$ and then using a PIR mechanism sends $i$ and requests the corresponding stored biometric template $b_{i}$. More precisely, $\mathcal{A S}$ sends to $\mathcal{D B}$ the encrypted value $\operatorname{Enc}\left(t_{j}\right)$, where $1 \leq j \leq N$ and $t_{j}=1$, if $j=i, 0$ otherwise.

- $\mathcal{D} \mathcal{B}$ computes: $\operatorname{Enc}\left(b_{i, k}\right)=\prod_{j=1}^{N} \operatorname{Enc}\left(t_{j}\right)^{b_{j, k}}$ where $1 \leq k \leq M$ and then sends the computed values $\operatorname{Enc}\left(b_{i, k}\right)$ to $\mathcal{A S}$.

Phase 3 - Communication $\mathcal{A S} \leftrightarrow \mathcal{M}$ :

- $\mathcal{A S}$ computes $v_{k}=\operatorname{Enc}\left(b_{i, k}^{\prime}\right) \operatorname{Enc}\left(b_{i, k}\right)=\operatorname{Enc}\left(b_{i, k}^{\prime} \oplus b_{i, k}\right)$, where $1 \leq k \leq$ $M$. Then, $\mathcal{A S}$ permutes $v_{k}$ and sends $\lambda_{k}=v_{\pi(k)}(1 \leq k \leq M)$ to $\mathcal{M}$.

- $\mathcal{M}$ decrypts the permuted vector $\lambda_{k}$ and checks whether the Hamming weight (HW) of the decrypted vector is less than a predefined threshold $\tau$. The result of this control is sent to $\mathcal{A S}$.

Phase 4 - Communication $\mathcal{A S} \rightarrow \mathcal{U}_{i}$ : Finally, $\mathcal{A S}$ accepts or rejects the authentication request (Out $\mathcal{A S}=1$ or Out $\mathcal{A S}=0$ respectively) depending on the value returned by $\mathcal{M}$. 


\section{Description of the Attacks}

Barbosa et al. [13] and Simoens et al. [14] presented several attacks on the above protocol when the adversary is a single entity or a combination of multiple entities. In addition, Simoens et al. [14 presented a framework for analysing security and privacy of biometric data in biometric authentication systems. In this section, we present a simple yet powerful algorithm (Algorithm 1) that can be used as a basis for a number of attacks. The attack algorithm takes a ciphertext as input and returns the corresponding plaintext by querying the matcher. The main enabler of this attack algorithm is the bit-by-bit encryption of the communication between the involved parties and the use of Hamming distance as the measure of whether the fresh biometric template matches the stored biometric profile. The algorithm uses as a subroutine the algorithm for the center search attack, but it is called only if the condition $\mathrm{HW}\left(b_{i}\right) \leq \tau$ holds; we urge the interested reader to consult Simoens et al. [14] for details on the attack

The Attack Idea Upon receiving from $\mathcal{A S}$ a vector $\lambda$ of ciphertexts, the matcher $\mathcal{M}$ first decrypts $\lambda$ component-by-component and then compares the Hamming weight of the resulting bitstring with a predefined threshold $\tau . \mathcal{M}$ responds YES to $\mathcal{A S}$ if the Hamming weight is less than $\tau$; otherwise, responds NO. Therefore, in order to find $b_{i}$ from $\lambda:=\operatorname{Enc}\left(b_{i}\right)=\left(\operatorname{Enc}\left(b_{i 1}\right), \operatorname{Enc}\left(b_{i 2}\right), \cdots, \operatorname{Enc}\left(b_{i M}\right)\right)$, an adversary (say, $\left.\mathcal{A S}\right)$ first finds a bitstring whose Hamming weight is equal to the threshold $\tau+1$ by repeatedly replacing the components of $(\operatorname{Enc}(0), \cdots, \operatorname{Enc}(0))$ with the corresponding components of $\lambda$ until it gets rejected by $\mathcal{M}$. By using this bitstring with Hamming weight $\tau+1$, the adversary is able to recover all bits of $b_{i}$ one by one, as shown in Algorithm 1 .

In the following attacks, we only consider the case when the authentication server $\mathcal{A S}$ (attacks 1 and 2) or the database $\mathcal{D B}$ (attack 3) is compromised, respectively.

Attack 1 - Compromised $\mathcal{A S}: \mathcal{A S}$ receives from $\mathcal{D B}$ the biometric reference template in encrypted form i.e. $\operatorname{Enc}\left(b_{i}\right)=c_{1}, \ldots, c_{M}$. Then, $\mathcal{A S}$ follows Algorithm 1. After executing the Algorithm 1, $\mathcal{A S}$ can successfully deduce all bits of $b_{i}$. The worst case complexity of this algorithm is $\max (2(\tau+M), 4 \tau+M)$, where $\tau$ is the threshold. We may note here that the complexity of the center search attack is $\max (2 \tau+M, 4 \tau)$ [14]. After executing this algorithm $\mathcal{A S}$ has successfully deduced $k$ out of the $M$ bits of $b_{i}$, where $M-k=\tau$ are the maximum allowed errors. By following a similar algorithm for the remaining $\tau$ bits, it can recover all bits of $b_{i}$. 
Attack 2 - Compromised $\mathcal{A S}$ : A variation of the previous attack can be performed if $\mathcal{A S}$ has also at his disposal a valid value $\operatorname{Enc}\left(b_{i}^{\prime} \oplus b_{i}\right)$. In this case Algorithm 1 can be executed twice: once for $\lambda=\operatorname{Enc}\left(b_{i}^{\prime}\right)$ and once for $\lambda=\operatorname{Enc}\left(b_{i}^{\prime} \oplus b_{i}\right)$. Thus, $\mathcal{A S}$ will be able to recover $b_{i}$ and $b_{i}^{\prime} \oplus b_{i}$ and subsequently $b_{i}^{\prime}$.

Attack 3 - Compromised $\mathcal{D B}:$ A variation of attack 1 can also be performed if $\mathcal{D B}$ is compromised. $\mathcal{D B}$ sets $\lambda=\operatorname{Enc}\left(t_{1}\right), \ldots, \operatorname{Enc}\left(t_{M}\right)$ if $M<N$; otherwise, $\lambda=\operatorname{Enc}\left(t_{1}\right), \ldots, \operatorname{Enc}\left(t_{N}\right), \operatorname{Enc}(0), \cdots, \operatorname{Enc}(0)$. This way, $\mathcal{D B}$ is able to recover $t_{j}$ 's by sending multiple queries to $\mathcal{M}$. Note that in the case of $M \leq N$, if it turns out that $t_{j}=0$, for all $j=1, \ldots, M$, then $\lambda$ can be chosen to be the encryption of the remaining $t_{j}$ 's. Here we remark that $\mathcal{D B}$ on its own cannot send queries to $\mathcal{M}$ directly. But since $\mathcal{M}$ does not check the integrity of received queries, the adversary can replace $\mathcal{A S}$ 's query to $\mathcal{M}$ with his own. In other words, here $\mathcal{D B}$ impersonates $\mathcal{A S}$ to $\mathcal{M}$.

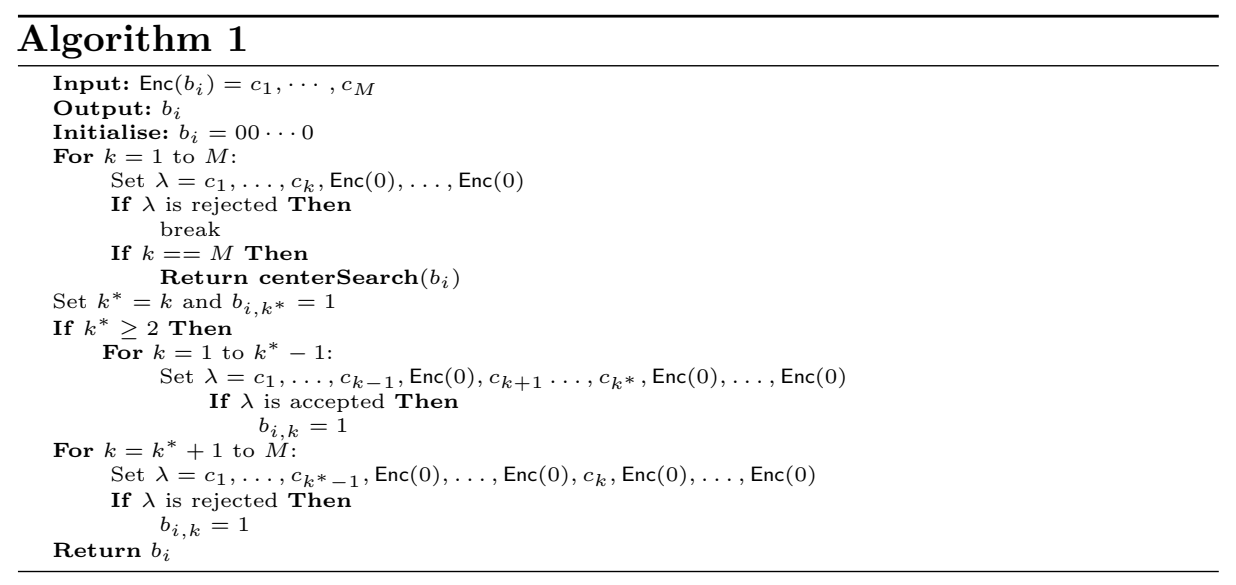

Thus, the Bringer et al. protocol is not secure or privacy-preserving in the malicious insider attack model. Because of the bit-by-bit encryption of the communication between the entities, the above presented attacks are straightforward and easy to mount. Plus, the complexity of the attacks is low. To mitigate the attacks, we next propose some modifications to the original protocol to improve its security and privacy preservation.

\section{Countermeasure}

Now, we propose modifications to the protocol under study to restore its security against the Attacks 1-3 presented in the previous section. Let us first discuss how we can protect the system against the Attack 1. We note that in this case the attacker has $\operatorname{Enc}\left(b_{i}\right)$ and wants to find out what $b_{i}$ is. 
If the matcher $\mathcal{M}$ does not directly compute the Hamming weight (HW) of the resulting bit-string from the decryption of the received ciphtertext, we may be able to protect the system against the Attack 1. So, in our modification, $\mathcal{M}$ shares two secret keys $K_{1}$ and $K_{2}$ with $\mathcal{S}$, a secret key $K_{3}$ with $\mathcal{A S}$, and two more secret keys $K_{4}$ and $K_{5}$ with $\mathcal{D} \mathcal{B}$. These keys are used for symmetric key schemes, therefore the length of these keys are not as long as the length of the key for the GM encryption. As before, pk and sk are $\mathcal{M}$ 's public and secret keys for GM encryption. $\mathcal{S}$ and $\mathcal{D B}$ also share a key $K_{\mathcal{S} \leftrightarrow \mathcal{D B}}$ that is used to derive a permutation $\pi$. In addition, $\mathcal{S}$ has a key $K$ that it uses to encrypt the user identity $\operatorname{ID}_{i}$.

During the enrollment phase, $\mathcal{S}$ stores $\left(b_{i}, i\right)$ at $\mathcal{D B}$ and $\left(\mathrm{id}_{i}, i\right)$, where $\mathrm{id}_{i}=\operatorname{Enc}_{K}\left(\mathrm{ID}_{i}\right)$ (a symmetric key encryption of $\operatorname{ID}_{i}$ with key $K$ ), at $\mathcal{A S}$.

The main changes take place in the authentication phase.

Phase 1 - Communication $\mathcal{U}_{i} \rightarrow \mathcal{S} \rightarrow \mathcal{A S}$ :

$-\mathcal{U}_{i} \rightarrow \mathcal{S}: \mathcal{U}_{i}$ provides a fresh biometric trait $b_{i}^{\prime}$ and $\mathrm{ID}_{i}$ to $\mathcal{S}$.

- $\mathcal{S} \rightarrow \mathcal{A S}: \mathcal{S}$ derives a permutation $\pi$ using the key $K_{\mathcal{S} \leftrightarrow \mathcal{D B}}$ (shared with $\mathcal{D B}$ ) and permutes $b_{i}^{\prime}$. Then, it generates two random bitstrings $S$ and $K_{1}^{\prime}$ of length $M$ and encrypts $\left(b_{i}^{\prime}\right)_{\pi} \oplus S$ with the public key pk (i.e. $a=\operatorname{Enc}\left(\left(b_{i}^{\prime}\right)_{\pi} \oplus S\right)$ ). In order to achieve forward security, $K_{1}^{\prime}$ is generated to replace $K_{1} . \mathcal{S}$ proceeds to compute $\omega=\operatorname{Enc}_{K_{1}}\left(S, K_{1}^{\prime}\right)$, an encryption of $S$ and $K_{1}^{\prime}$ with $K_{1}$, and computes $\sigma=\operatorname{TAG}\left(\omega, K_{2}\right)$. Also, $\mathcal{S}$ replaces $K_{1}$ with $K_{1}^{\prime}$, which will be used in the next run of the protocol and deletes $K_{1}$ permanently. Finally, $\mathcal{S}$ sends $a$ and $(\omega, \sigma)$ along with the encryption of the claimed identity $\mathrm{id}_{i}=\mathrm{Enc}_{K}\left(\mathrm{ID}_{i}\right)$ to $\mathcal{A S}$. Note that this encryption of $\mathrm{ID}_{i}$ is done to protect it from an adversary observing the communication from $\mathcal{S}$ to $\mathcal{A S}$.

Phase 2 - Communichtion $\mathcal{A S} \leftrightarrow \mathcal{D} \mathcal{B}$ :

- $\mathcal{A S} \rightarrow \mathcal{D B}: \mathcal{A S}$ extracts the index $i$ from id $i_{i}$ and sends $d_{j}=\operatorname{Enc}\left(t_{j}\right)$ to $\mathcal{D B}$, for $j=1, \cdots, N$, where $t_{j}$ is the same as before.

- $\mathcal{D B} \rightarrow \mathcal{A S}: \mathcal{D B}$ derives $\pi$ from $K_{\mathcal{S} \leftrightarrow \mathcal{D B}}$, generates two random bitstrings $S^{\prime}$ and $K_{4}^{\prime}$ of length $M$, and computes $c_{k}=\operatorname{Enc}\left(\left(b_{i, k}\right)_{\pi} \oplus S_{k}^{\prime}\right)=$

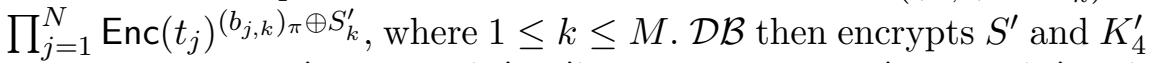
using $K_{4}$ to get $\omega^{\prime}=\operatorname{Enc}_{K_{4}}\left(S^{\prime}, K_{4}^{\prime}\right)$, and computes $\sigma^{\prime}=\operatorname{TAG}\left(\omega^{\prime}, K_{5}\right)$. After that, $\mathcal{D B}$ replaces $K_{4}$ with $K_{4}^{\prime}$ (to guarantee forward security) and deletes $K_{4}$. Finally, $\mathcal{D B}$ sends $c,\left(\omega^{\prime}, \sigma^{\prime}\right)$ to $\mathcal{A S}$.

Phase 3 - Communication $\mathcal{A S} \leftrightarrow \mathcal{M}$ :

- $\mathcal{A S} \rightarrow \mathcal{M}: \mathcal{A S}$ computes $\lambda_{k}=a_{k} c_{k}=\operatorname{Enc}\left(\left(b_{i, k}^{\prime}\right)_{\pi} \oplus S\right) \operatorname{Enc}\left(\left(b_{i, k}\right)_{\pi} \oplus\right.$ $\left.S^{\prime}\right)=\operatorname{Enc}\left(\left(b_{i, k}^{\prime} \oplus b_{i, k}\right)_{\pi} \oplus S \oplus S^{\prime}\right)$, for $1 \leq k \leq M$, computes $\sigma^{\prime \prime}=$ $\operatorname{TAG}\left(\lambda, K_{3}\right)$, and sends $(\omega, \sigma),\left(\omega^{\prime}, \sigma^{\prime}\right)$, and $\left(\lambda, \sigma^{\prime \prime}\right)$ to $\mathcal{M}$. 
$-\mathcal{M} \rightarrow \mathcal{A S}: \mathcal{M}$ first checks the authenticity of $\omega, \omega^{\prime}$ and $\lambda$ by respectively running $\operatorname{VRFY}\left(\omega, \sigma, K_{2}\right), \operatorname{VRFY}\left(\omega^{\prime}, \sigma^{\prime}, K_{5}\right)$, and $\operatorname{VRFY}\left(\lambda, \sigma^{\prime \prime}, K_{3}\right)$. If any one of them is not authentic, it outputs $\perp$ (i.e. aborts the protocol). Otherwise, it proceeds to obtain $S, K_{1}^{\prime} \leftarrow \operatorname{Dec}_{K_{1}}(\omega), S^{\prime}, K_{4}^{\prime} \leftarrow$ $\operatorname{Dec}_{K_{4}}\left(\omega^{\prime}\right)$, and $\left(b_{i}^{\prime} \oplus b_{i}\right)_{\pi} \leftarrow \operatorname{Dec}(\lambda) \oplus S \oplus S^{\prime}$; and replaces $K_{1}$ and $K_{4}$ with $K_{1}^{\prime}$ and $K_{4}^{\prime}$, respectively. Lastly, $\mathcal{M}$ checks whether the $\mathrm{HW}\left(\left(b_{i}^{\prime} \oplus b_{i}\right)_{\pi}\right) \leq \tau$ and sends the result of this control to $\mathcal{A S}$.

Phase 4 - Communication $\mathcal{A S} \rightarrow \mathcal{U}_{i}$ : Finally, $\mathcal{A S}$ accepts or rejects the authentication request $\left(\mathrm{Out}_{\mathcal{A S}}=1\right.$ or Out $_{\mathcal{A S}}=0$ respectively) depending on the value returned by $\mathcal{M}$.

We should note here that the reason for replacing $K_{1}$ and $K_{4}$ with new independently generated $K_{1}^{\prime}$ and $K_{4}^{\prime}$, respectively, was to ensure forward security and thus to limit the damage in case the keys are compromised. The main question we want to answer now is: How secure is the improved protocol against the presented attacks? We address this question in the following section.

\section{Security Analysis}

Let us assess the security of the modified protocol. Before we proceed, we recall that we aim for security in the malicious, but non-colluding model, meaning that any entity can deviate from the protocol specifications but none of the entities may collude with each other. Therefore, we focus on security against malicious insider attacks. Since our primary goal is to assure security and privacy of biometric templates and user identity, we do not consider denial of service type of attacks in our analysis.

To begin with, let us analyse case-by-case what may happen when the entities, except for the sensor $\mathcal{S}$ which we assume to be honest and cannot be compromised, are malicious.

- Attacker $=\mathcal{A S}: \mathcal{A S}$ has knowledge of $K_{3}$, so it can send arbitrary queries to $\mathcal{M}$. In addition, it has at its disposal the encrypted user identity $\mathrm{id}_{i}=\operatorname{Enc}_{K}\left(\operatorname{ID}_{i}\right)$, encrypted biometric templates $\operatorname{Enc}\left(\left(b_{i}^{\prime}\right)_{\pi} \oplus S\right)$ and $\operatorname{Enc}\left(\left(b_{i}\right)_{\pi} \oplus S^{\prime}\right), \omega=\operatorname{Enc}_{K_{1}}\left(S, K_{1}^{\prime}\right), \omega^{\prime}=\operatorname{Enc}_{K_{4}}\left(S^{\prime}, K_{4}^{\prime}\right)$, their authentication tags $\sigma=h_{K_{2}}(\omega), \sigma^{\prime}=h_{K_{5}}\left(\omega^{\prime}\right)$. He wants to use all this information to gain knowledge of $b_{i}^{\prime}, b_{i}$, and the linkage between $\mathrm{ID}_{i}$ and a biometric template $b_{i}$. It may arbitrarily deviate from the protocol specifications, except that it is not allowed to compromise or collude with another protocol entity. Note that $\mathcal{A S}$ can always cause denial of service to legitimate users by providing wrong input to $\mathcal{M}$.

- Attacker $=\mathcal{D B}: \mathcal{D B}$ has knowledge of all stored biometric templates and of $K_{4}, K_{5}, S^{\prime}$ and $\pi$. However, it does not know which $b_{i}$ is 
related to which user identity $\mathcal{U}_{i}$. It also does not know which user is attempting to authenticate himself to the server $\mathcal{A S}$. Therefore, its goal is to learn which user is trying to authenticate himself and to which user a biometric template belongs. It may also deviate from the protocol specifications, but it cannot collude with other entities.

- Attacker $=\mathcal{M}: \mathcal{M}$ has the secret keys sk, $K_{1}, K_{2}, K_{3}, K_{4}$, and $K_{5}$. Its goal is to distinguish whether two authentication attempts are from the same user. Since we assume that communications between the entities cannot be eavesdropped, it cannot use the secret keys to learn $b_{i}$ and $b_{i}^{\prime}$, unless it colludes with $\mathcal{A S}$.

The modified protocol is secure and preserves the privacy of biometric templates and user identity. In particular, none of the entities $\mathcal{A S}, \mathcal{D B}$, and $\mathcal{M}$, all malicious but non-colluding and PPT, can link a biometric template to a user identity and a malicious $\mathcal{D B}$ cannot distinguish whether two authentication attempts are from the same user. More precisely, Theorem 1 and Theorem 2 stated in Bringer et al. [1] also hold in the proposed modified protocol. We provide their proofs for the modified protocol in Appendix B. Finally, the modified proposed protocol is secure against malicious authentications servers $\mathcal{A S}$ as stated in the following theorem (we provide its proof in Appendix C).

Theorem 1. If the Assumptions 1-3 hold and if (a) $S$ and $S^{\prime}$ are generated using $\epsilon$-secure PNGs (cf. Definition 3), (b) the symmetric encryption schemes SKE used between the sensor $\mathcal{S}$ and the matcher $\mathcal{M}$, and between the database $\mathcal{D B}$ and the matcher $\mathcal{M}$, is IND-COA-secure, and (c) the GM scheme is IND-CPA-secure. Then, our modified protocol is secure against any malicious authentication server $\mathcal{A S}$.

Forward Security Informally, forward security means that the disclosure of a secret key material does not compromise the secrecy of the exchanged communications from previous rounds. As we briefly mentioned in the previous section, our modified protocol has forward security. In particular, the biometric templates exchanged will not be affected by a future disclosure of the secret key used to encrypt them. The original protocol, on the other hand, does not provide forward security. This is because if the matcher $\mathcal{M}$ 's secret key is compromised, then all biometric templates exchanged in the past can be learned. But in the modified protocol, the adversary learns the biometric templates in the present round (and onwards) only.

Comparison In comparison with the original protocol, in our modification each protocol entity performs additional cryptographic com- 
putations such as, symmetric key encryption/decryption, MAC generation/verification, and generation of pseudo-random numbers. In particular, in the case of $\mathcal{S}$, in the original protocol, $\mathcal{S}$ only computes the encryption of the fresh biometric samples using the GM encryption. But in the modified protocol, in addition to that, $\mathcal{S}$ first generates $S, K_{1}^{\prime}$ and then computes $\omega=\operatorname{Enc}_{K_{1}}\left(S, K_{1}^{\prime}\right)$ and $\mathrm{Enc}_{K}\left(\mathrm{ID}_{i}\right)$ using a symmetric encryption and computes an authentication tag for $\omega$ using a suitable MAC. In the case of $\mathcal{A S}$, the only additional computation done in the modified protocol is the authentication tag generation for $\lambda$, i.e. $\sigma^{\prime \prime}=h_{K_{3}}(\lambda)$. In the case of $\mathcal{D B}$, in the modified protocol, $\mathcal{D B}$ first generates $S^{\prime}, K_{4}^{\prime}$ and then $\omega^{\prime}=\operatorname{Enc}_{K_{4}}\left(S^{\prime}, K_{4}^{\prime}\right)$, $\sigma^{\prime}=\mathrm{TAG}_{K_{5}}\left(\omega^{\prime}\right)$. In the case of $\mathcal{M}$, in the modified protocol, the additional computations done by $\mathcal{M}$ are: $\operatorname{VRFY}\left(\omega, \sigma, K_{2}\right), \operatorname{VRFY}\left(\omega^{\prime}, \sigma^{\prime}, K_{5}\right)$, $\operatorname{VRFY}\left(\lambda, \sigma^{\prime \prime}, K_{3}\right), \operatorname{Dec}_{K_{1}}(\omega)$ and $\operatorname{Dec}_{K_{4}}\left(\omega^{\prime}\right)$. Also, it XORs $S$ and $S^{\prime}$ with $\operatorname{Dec}(\lambda)$. It is evident that in the modified protocol, each system entity performs some additional computations than required in the original protocol. However, as they are symmetric cryptographic operations, these computations are not as heavy as those done in the GM encryption.

\section{Conclusions}

We investigated the security of a privacy-preserving biometric authentication protocol proposed by Bringer et al. that uses the Goldwasser-Micali cryptosystem in the malicious attack model. We presented a simple attack algorithm that can be employed to mount a number of attacks on the system to either obtain the reference biometric template $\left(b_{i}\right)$ or the identity $\left(\mathrm{ID}_{i}\right)$ of a user associated with a biometric template $\left(b_{i}\right)$. Furthermore, we proposed an improved version of the Bringer et al. [1] protocol and proved its security against malicious, but non-colluding insider attacks. As future work, we would like to investigate how to achieve security and privacy against colluding internal adversaries.

\section{Acknowledgements}

We would like to thank Serge Vaudenay for suggesting the attack algorithm and Julien Bringer for the helpful discussions. This work was partially supported by the FP7-STREP project "BEAT: Biometric Evaluation and Testing", grant number: 284989.

\section{References}

1. Bringer, J., Chabanne, H., Izabachène, M., Pointcheval, D., Tang, Q., Zimmer, S.: An application of the Goldwasser-Micali cryptosystem to biometric authentication. In: ACISP'07. LNCS (2007) 96-106 
2. Ouafi, K., Vaudenay, S.: Strong Privacy for RFID Systems from Plaintext-Aware Encryption. In: CANS'12. Volume 7712 of LNCS. Springer (2012) 247-262

3. Vaudenay, S.: On Privacy Models for RFID. In: ASIACRYPT'07. Volume 4833 of LNCS., Springer (2007) 68-87

4. Hermans, J., Pashalidis, A., Vercauteren, F., Preneel, B.: A new RFID privacy model. In: ESORICS'11. LNCS (2011) 568-587

5. Juels, A., Weis, S.A.: Authenticating pervasive devices with human protocols. In: Proceedings of the 25th annual international conference on Advances in Cryptology. CRYPTO'05 (2005) 293-308

6. Gilbert, H., Robshaw, M.J.B., Sibert, H.: Active attack against HB+: a provably secure lightweight authentication protocol. Electronic Letters 41 (2005) 1169-1170

7. Penrose, L.: Dermatoglyphic topology. Nature 205 (1965) 544-546

8. Bolling, J.: A window to your health. Jacksonville Medicine, Special Issue: Retinal Diseases 51 (2000)

9. Rivest, R.L., Adleman, L., Dertouzos, M.L.: On data banks and privacy homomorphisms. In: Foundations of Secure Computation. Academic Press (1978) $165-179$

10. Rabin, M.O.: How to exchange secrets with oblivious transfer. Technical Report TR-81, Aiken Computation Lab, Harvard University (1981)

11. Even, S., Goldreich, O., Lempel, A.: A randomized protocol for signing contracts. Commun. ACM 28(6) (1985) 637-647

12. Goldwasser, S., Micali, S.: Probabilistic encryption \& how to play mental poker keeping secret all partial information. In: Proceedings of ACM symposium on Theory of computing. STOC '82 (1982) 365-377

13. Barbosa, M., Brouard, T., Cauchie, S., Sousa, S.M.: Secure Biometric Authentication with Improved Accuracy. In: ACISP'08. LNCS (2008) 21-36

14. Simoens, K., Bringer, J., Chabanne, H., Seys, S.: A framework for analyzing template security and privacy in biometric authentication systems. IEEE Transactions on Information Forensics and Security 7(2) (2012) 833-841

15. Bringer, J., Chabanne, H.: An authentication protocol with encrypted biometric data. In: AFRICACRYPT'08. LNCS (2008) 109-124

16. Lipmaa, H.: An oblivious transfer protocol with log-squared communication. In ISC. Volume 3650 of LNCS. (2005) 314-328

17. Stoianov, A.: Cryptographically secure biometrics. SPIE 7667, Biometric Technology for Human Identification VII 76670C (2010) 76670C-76670C-12

18. Blum, M., Goldwasser, S.: An efficient probabilistic public key encryption scheme which hides all partial information. In: CRYPTO'84. (1985) 289-299

19. Menezes, A., van Oorschot, P.C., Vanstone, S.A.: Handbook of Applied Cryptography. CRC Press (1996)

\section{A Appendix}

$\operatorname{Exp}_{\mathrm{SKE}, \mathcal{A}}^{\mathrm{IND}-\mathrm{COA}}$ is the IND-COA game against an SKE scheme defined as follows.

$$
\begin{array}{|rl}
\operatorname{Exp}_{\mathrm{SKE}, \mathcal{A}}^{\text {IND-COA }: K} & \leftarrow \operatorname{KeyGen}\left(1^{\ell}\right) \\
m_{0}, m_{1} & \leftarrow A_{1}\left(1^{\ell}\right) \\
c & \leftarrow \operatorname{Enc}\left(m_{\beta}, K\right), \beta \stackrel{R}{\leftarrow}\{0,1\} \\
\beta^{\prime} & \leftarrow A_{2}\left(m_{0}, m_{1}, c\right) \\
\text { Return 1 if } \beta^{\prime}=\beta, 0 \text { otherwise } &
\end{array}
$$


The adversary's advantage in this game is defined as $\operatorname{Adv}_{\mathrm{SKE}, \mathcal{A}}^{\mathrm{IND}-\mathrm{COA}}=$ $\left|2 \operatorname{Pr}\left(\operatorname{Exp}_{\mathrm{SKE}, \mathcal{A}}^{\mathrm{IND}-\mathrm{COA}}=1\right)-1\right|$. A SKE scheme is said to be IND-COAsecure, if $\forall$ PPT adversary $\mathcal{A}, \operatorname{Adv}_{\mathrm{SKE}, \mathcal{A}}^{\mathrm{IND}-\mathrm{COA}} \leq \operatorname{neg}(\ell)$, where (and below) $\operatorname{negl}(\ell): \mathbf{N} \mapsto[0,1]$ is a negligible function meaning that for all positive polynomials $P$ and all sufficiently large $\ell \in \mathbf{N}$, we have $\operatorname{neg}(\ell)<1 / P(\ell)$.

$\operatorname{Exp}_{\mathrm{GM}, \mathcal{A}}^{\mathrm{IND}-\mathrm{CPA}}$ is the IND-CPA game against the GM encryption and is defined as in the previous game, but now the adversary has access to the public key. This scheme is said to be IND-CPA secure if $\forall$ PPT adversary $\mathcal{A}, \operatorname{Adv}_{\mathrm{GM}, \mathcal{A}}^{\mathrm{IND}-\mathrm{CPA}}=\left|2 \operatorname{Pr}\left(\operatorname{Exp}_{\mathrm{GM}, \mathcal{A}}^{\mathrm{IND}-\mathrm{CPA}}=1\right)-1\right| \leq \operatorname{negl}(\ell)$.

\section{B Appendix}

Here we prove the Theorem 1 and 2 in Bringer et al. [1 in the case of our improved protocol.

Theorem 2. For any $I D_{i_{0}}$ and two biometric templates $b_{i_{0}}^{\prime}, b_{i_{1}}^{\prime}$, where $i_{0}, i_{1} \geq 1$ and $b_{i_{0}}^{\prime}$ is the biometric template related to $I D_{i_{0}}$, any of the malicious, but not colluding $\mathcal{A S}, \mathcal{D B}$, and $\mathcal{M}$ can only distinguish between $\left(I D_{i_{0}}, b_{i_{0}}^{\prime}\right)$ and $\left(I D_{i_{0}}, b_{i_{1}}^{\prime}\right)$ with a negligible advantage.

Proof. Since $\mathcal{D B}$ and $\mathcal{M}$ have no access to user identities, their advantage is 0 in distinguishing between $\left(\mathrm{ID}_{i_{0}}, b_{i_{0}}^{\prime}\right)$ and $\left(\mathrm{ID}_{i_{0}}, b_{i_{1}}^{\prime}\right)$.

In the case of $\mathcal{A S}$, it has access to id $i_{0}=\operatorname{Enc}_{K}\left(\operatorname{ID}_{i_{0}}\right)$, where $\operatorname{Enc}_{K}(\cdot)$ is a symmetric encryption with the sensor $\mathcal{S}$ 's key $K$. However, even if $\mathcal{A S}$ knows $\mathrm{ID}_{i_{0}}$, it cannot distinguish between $\left(\mathrm{ID}_{i_{0}}, b_{i_{0}}^{\prime}\right)$ and $\left(\mathrm{ID}_{i_{0}}, b_{i_{1}}^{\prime}\right)$, except with a negligible probability, as we see below.

Suppose that $\mathcal{A S}$ has a non-negligible advantage in distinguishing between $\left(\mathrm{ID}_{i_{0}}, b_{i_{0}}^{\prime}\right)$ and $\left(\mathrm{ID}_{i_{0}}, b_{i_{1}}^{\prime}\right)$. Then, we can construct an adversary $\mathcal{A}$, consisting of algorithms $A_{1}$ and $A_{2}$, such that $\mathcal{A}$ 's advantage in the following game is non-negligible, contradicting the IND-CPA-security of GM cryptosystem:

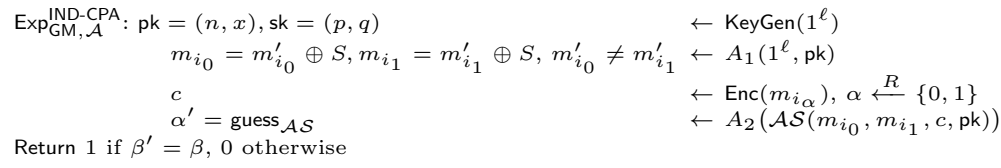

In the experiment, $A_{2}$ simulates the biometric authentication protocol by letting pk be $\mathcal{M}$ 's public key and storing $m_{i_{0}}^{\prime}$ and $m_{i_{1}}^{\prime}$ in $\mathcal{D} \mathcal{B} . A_{2}$ then asks $\mathcal{A S}$ to guess $\beta$ from $c=\operatorname{Enc}\left(m_{i_{\beta}}\right)=\operatorname{Enc}\left(m_{i_{\beta}}^{\prime} \oplus S\right)$ and returns $\beta$ as the guess for $\alpha$. So, $\mathcal{A}$ wins if $\mathcal{A S}$ wins in his guess. Thus, $\mathcal{A S}$ can only distinguish between $\left(\mathrm{ID}_{i_{0}}, b_{i_{0}}^{\prime}\right)$ and $\left(\mathrm{ID}_{i_{0}}, b_{i_{1}}^{\prime}\right)$ with negligible probability. 
The next theorem shows that a malicious database $\mathcal{D B}$ cannot distinguish whether two authentication attempts are from the same user.

Theorem 3. For any two users $\mathcal{U}_{i_{0}}$ and $\mathcal{U}_{i_{1}}$, where $i_{0}, i_{1} \geq 1$, if $\mathcal{U}_{i_{\beta}}$ where $\beta \in\{0,1\}$ makes an authentication attempt, then the malicious $\mathcal{D} \mathcal{B}$ can only guess $\beta$ with a negligible advantage. Here, the adversary's advantage is defined as $\left|\operatorname{Pr}\left\{\beta=\beta^{\prime}\right\}-1 / 2\right|$, where $\beta^{\prime}$ is $\mathcal{D B}$ 's guess.

Proof (of Theorem 3). DB guesses $\beta$ from $\operatorname{Enc}\left(t_{j}\right)$, for $j=1, \cdots, N$, where $t_{j}=1$ when $j=i_{\beta}(\beta \in\{0,1\})$, otherwise $t_{j}=0$. The proof is similar to that of Theorem 2 in Bringer et al. [1].

Suppose that $\mathcal{D B}$ can guess $\beta$ with non-negligible advantage. Then, we can construct a PPT adversary $\mathcal{A}$, consisting of $A_{1}$ and $A_{2}$, that uses $\mathcal{D B}$ as a blackbox to win in the following game with non-negligible advantage; contradicting the IND-CPA-security of GM cryptosystem:

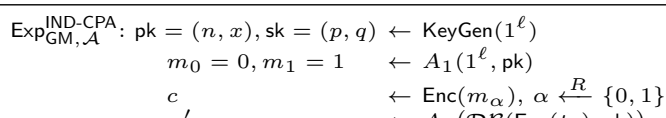

where $\operatorname{Enc}\left(t_{i_{1}}\right)=c, \operatorname{Enc}\left(t_{i_{0}}\right)=y^{2} x c, y \stackrel{R}{\leftarrow} \mathbb{Z}_{n}^{\star}, t_{j}=0, \forall j \neq i_{0}, i_{1}$. Note that if $c=\operatorname{Enc}\left(m_{0}\right)$, then $y^{2} x c$ is not a quadratic residue $\bmod n$, so $\mathcal{D B}$ 's guess, which is 0 , and $\alpha$ coincide. Similarly, if $c=\operatorname{Enc}\left(m_{1}\right)$, then $y^{2} x c$ is a quadratic residue $\bmod n$, so $\mathcal{D B}$ 's guess, which is 1 , and $\alpha$ coincide. Hence, $\mathcal{D B}$ 's advantage of guessing $\beta$ correctly should be negligible.

\section{Appendix}

Here, we present the proof of Theorem 1 . Let $\mathcal{A}$ be any PPT adversary, consisting of two algorithms $A_{1}$ and $A_{2}$. Let us consider the following game against the modified biometric authentication protocol $\Pi$. Let KeyGen be an algorithm that generates both symmetric and asymmetric keys needed in the protocol, upon $1^{\ell}$ (a string of 1 s of length $\ell$ ) as an input. As usual, $\ell$ is a security parameter.

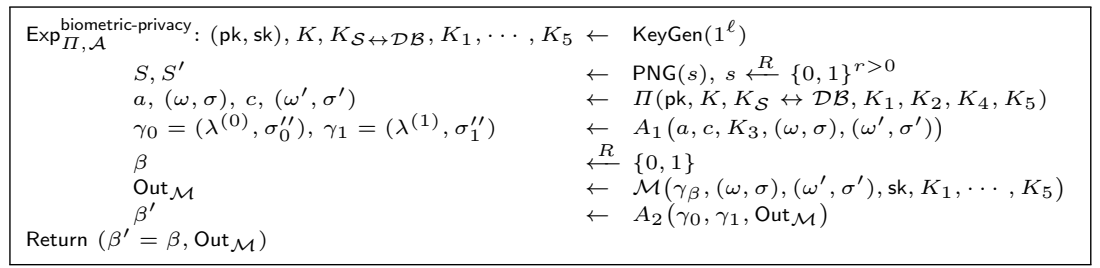


The adversary's advantage $\operatorname{Adv}_{\Pi, \mathcal{A}}^{\text {biometric-privacy }}$ at the end of this game is defined as $\operatorname{Adv}_{\Pi, \mathcal{A}}^{\text {biometric-privacy }}=\left|\operatorname{Pr}\left\{\beta^{\prime}=\beta\right\}-1 / 2\right|$, where $\beta \in\{0,1\}$ is $\mathcal{M}$ 's choice and $\beta^{\prime}$ is the adversary's guess for $\beta$. We say that the biometric authentication protocol is secure against malicious $\mathcal{A S}$, if $\operatorname{Adv}_{\Pi, \mathcal{A}}^{\text {biometric-privacy }} \leq \operatorname{negl}(\ell)$.

Note that as stated in Assumption 2, we assume that the adversary does not have access to an acceptable biometric template, because otherwise the adversary can easily produce two challenges so that it wins the above experiment with non-negligible advantage.

Proof (of Theorem 1). Case 1. $\mathrm{HW}\left(\operatorname{Dec}\left(\lambda^{(\beta)}\right) \oplus S \oplus S^{\prime}\right) \leq \tau$, for $\forall \beta \in$ $\{0,1\}$. In this case, $\mathcal{M}$ 's output always be the same (i.e., Out $\mathcal{M}=$ YES.) Hence, the adversary's advantage in this case is 0 .

Case 2. $\mathrm{HW}\left(\operatorname{Dec}\left(\lambda^{(\beta)}\right) \oplus S \oplus S^{\prime}\right)>\tau$, for $\forall \beta \in\{0,1\}$. Also in this case, $\mathcal{M}$ 's output always be the same (i.e., Out $\mathcal{M}=$ NO). Hence, the adversary's advantage in this case is 0 .

Case 3. $\operatorname{HW}\left(\operatorname{Dec}\left(\lambda^{(\beta)}\right) \oplus S \oplus S^{\prime}\right) \leq \tau$ and $\operatorname{HW}\left(\operatorname{Dec}\left(\lambda^{(1-\beta)}\right) \oplus S \oplus S^{\prime}\right)>$ $\tau$. Suppose that a PPT adversary $\mathcal{A}$ has a non-negligible advantage $\delta$ of winning the game $\operatorname{Exp}_{\Pi, \mathcal{A}}^{\text {biometric-privacy }}$. Then we can construct a PPT adversary $\overline{\mathcal{A}}$ that wins in $\operatorname{Exp}_{\mathrm{SKE}, \overline{\mathcal{A}}}^{\mathrm{IND}-\mathrm{COA}}$ and/or $\operatorname{Exp}_{\mathrm{GM}, \overline{\mathcal{A}}}^{\mathrm{IND}-\mathrm{CPA}}$ with advantage $\delta$. The construction of such an adversary $\overline{\mathcal{A}}$, for example in the case of $\operatorname{Exp}_{\mathrm{SKE}, \overline{\mathcal{A}}}^{\mathrm{IND}-\mathrm{COA}}$, may proceed as follows:

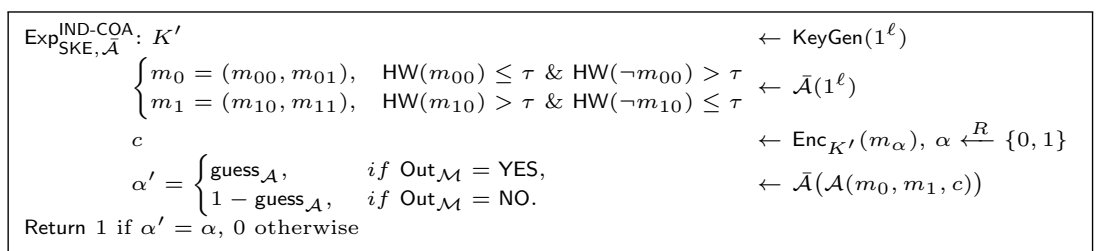

where $\left|m_{00}\right|=\left|m_{10}\right|=|S|$ and $\left|m_{01}\right|=\left|m_{11}\right|=\left|K_{1}\right| \cdot \overline{\mathcal{A}}$ then simulates the biometric authentication protocol and replaces, without loss of generality, the symmetric key encryption scheme between the sensor $\mathcal{S}$ and the matcher $\mathcal{M}$. More precisely, $\overline{\mathcal{A}}$ replaces $\omega$ with the challenge ciphertext $c$ and $\omega^{\prime}$ with an encryption of a bitstring of all zeros. $\overline{\mathcal{A}}$ then runs $A_{1}$ to obtain $\gamma_{0}=\left(\operatorname{Enc}_{\mathrm{GM}}(0), \sigma_{0}^{\prime \prime}\right)$ and $\gamma_{1}=\left(\operatorname{Enc}_{\mathrm{GM}}(1), \sigma_{1}^{\prime \prime}\right)$, where 0 and 1 respectively stand for bitstrings of all zeros and ones. If Out $\mathcal{M}_{\mathcal{M}}=\mathrm{YES}, \overline{\mathcal{A}}$ outputs $\mathcal{A}^{\prime}$ 's guess $\beta^{\prime}$ as $\alpha^{\prime}$; if Out $\mathrm{M}_{\mathcal{M}}=\mathrm{NO}, \overline{\mathcal{A}}$ outputs $1-\beta^{\prime}$ as $\alpha^{\prime}$. This is because, when Out w $_{\mathcal{M}}=$ YES, $\beta^{\prime}=0$ would indicate that $\operatorname{HW}\left(m_{\alpha 0}\right) \leq \tau$, and $\beta^{\prime}=1$ would indicate that $\operatorname{HW}\left(1 \oplus m_{\alpha 0}\right)>\tau$. And similarly, when Out $_{\mathcal{M}}=\mathrm{NO}, \beta^{\prime}=0$ would indicate that $\operatorname{HW}\left(m_{\alpha 0}\right)>\tau$, and $\beta^{\prime}=1$ would indicate that $\operatorname{HW}\left(1 \oplus m_{\alpha 0}\right) \leq \tau$. Hence, if $\mathcal{A}$ wins, so does $\overline{\mathcal{A}}$. 